\title{
Analyse und Optimierung von Lehrdienstleistungen mittels Service Blueprinting
}

\author{
Konzeption und erste empirische Befunde \\ René Wegener, Philipp Menschner, Jan Marco Leimeister \\ Fachgebiet Wirtschaftsinformatik, Universität Kassel
}

\begin{abstract}
Wir stellen einen Ansatz zur Analyse und produktivitätsorientierten Gestaltung universitärer Lehre vor. Die Zielsetzung liegt dabei in einer Produktivitätssteigerung der Lehre durch Erhöhung des Outputs (Zufriedenheit und Lernerfolg) bei gleichzeitiger Reduzierung des Inputs (Lehraufwand). Hierzu wurde die aus dem Service Engineering bekannte Methode des Service Blueprinting eingesetzt, um den Lehrerbringungsprozess zu modellieren und Teilaktivitäten auf Möglichkeiten stärkerer Studierendenintegration sowie intelligenter Teilautomatisierung hin zu prüfen. Als erstes Anwendungsbeispiel wurden die Studierenden in die besonders aufwändige Erstellung vorlesungsergänzender multimedialer Selbstlerneinheiten eingebunden. Bisherige Evaluationsergebnisse deuten darauf hin, dass diese für den Lehrenden ressourcenschonendere Vorgehensweise nicht zu Lasten der Studierenden geht, sondern eine Bereicherung des Lernerlebnisses darstellt. Dieser Ansatz ist neuartig für die Entwicklung von Lernszenarien, da er neben Outputfaktoren auch den Input berücksichtigt und somit zu einer erhöhten Produktivität der universitären Lehre beitragen kann.
\end{abstract}

\section{Einleitung}

„Das Hochschulsystem leistet einen wichtigen, langfristig wirksamen Beitrag zur Förderung von Wachstum und Innovation - und damit zur Bewältigung der Herausforderungen des demographischen und wirtschaftlichen Wandels" (Wissenschaftsrat 2006, S. 8). Diese Aussage des Wissenschaftsrats zeigt, dass die hochschulische Bildung wesentlich zur Zukunftsfähigkeit der Bundesrepublik Deutschland beiträgt. Auch die OECD bestätigt, dass Bildungsinvestitionen sich auf die Produktivität der Wirtschaft eines Landes auswirken und dadurch einen hohen 
Return on Investment besitzen (OECD 2003, S. 17). Dennoch leidet das deutsche Hochschulsystem unter anhaltender Unterfinanzierung (Wissenschaftsrat 2006, S. 6). Diese Problematik verschärft sich insbesondere, da die Studierendenzahlen in den nächsten Jahren deutlich steigen werden, auf eine Zahl von bis zu 450.000 Studienanfängern im Jahr 2012 (Kultusministerkonferenz 2005, S. 29). Während es 2004 noch ca. 1,95 Millionen Studierende an deutschen Hochschulen gab, beläuft sich eine Prognose für 2012 auf bis zu über 2,6 Millionen (Kultusministerkonferenz 2005, S. 25+). Da bereits 2003 auf einen Professor fast 150 Studierende fielen (Wissenschaftsrat 2006, S. 52), steht die hochschulische Dienstleistung Lehre aufgrund dieser Zahlen vor einer gewaltigen Aufgabe. Der deutsche Wissenschaftsrat sieht daher für die hochschulische Ausbildung einen hohen Bedarf nach Steigerung von Effizienz und Effektivität (Wissenschaftsrat 2006, S. 72f.). Schließlich ist die Ausbildung eines Studierenden auch sehr kostspielig - für einen Masterstudenten an einer Universität betrugen die Ausbildungskosten 2006 pro Universität durchschnittlich 47.400 Euro (Brugger et al. 2009, S. 43).

In Anbetracht dieser Zahlen erscheint die klassische Vorlesung ohne nennenswerte Interaktion mit den Studierenden aus Gründen der Effizienz teilweise alternativlos, zumal ein Mehr an Interaktion für gewöhnlich auch mit einem deutlich höherem Aufwand verbunden ist. Hochwertige, individualisierte Lernangebote können daher nur umgesetzt werden, wenn sie auch unter wirtschaftlichen Gesichtspunkten zufriedenstellend sind.

Um diesem Spannungsverhältnis zwischen individueller, hochwertiger Lernerfahrung einerseits und Wirtschaftlichkeit andererseits zu begegnen, wird in diesem Artikel auf Erkenntnisse aus dem Service Engineering zurückgegriffen. Dieses liefert Methoden zur systematischen Entwicklung von Dienstleistungen. Die Zielsetzung liegt dabei in einer Steigerung der Produktivität von Lehrdienstleistungen.

Als konkrete Methode wird das in der Dienstleistungsforschung bereits vielseitig eingesetzte Konzept des Service Blueprinting aufgegriffen. Dieses wird zunächst erläutert und auf den Problembereich hochschulischer Lehre übertragen. Ausgehend von diesem Begriffsverständnis wird die Methode des Blueprinting dann auf ein typisches Lehr-Lern-Angebot mit Blended Learning Charakter angewendet. Dabei wird aufgezeigt, wo Potenziale zur Optimierung des Lehrerbringungsprozesses liegen. Anhand eines Beispiels wird gezeigt, wie diese didaktisch fundiert realisiert werden können. Erste empirische Befunde zeigen dabei das Potenzial dieses Vorgehens auf.

Der hier vorgestellte Ansatz ist neu, weil er das pädagogisch motivierte Streben nach maximalem Lernerfolg mit der Perspektive des kostenorientierten Bildungscontrollings verbindet und so eine ganzheitliche Betrachtungsweise darstellt. Dieser Artikel liefert somit konzeptionelle Implikationen für die universitäre Ausbildung, welche einen Beitrag dazu leisten können, die Produktivität der Dienstleistung Lehre an der Universität zu steigern. 


\section{Hintergrund und Definitionen}

\subsection{Bildung als Dienstleistung}

Für Dienstleistungen oder Services existieren unterschiedlichste Definitionen, je nach Tätigkeitsbereich oder Forschungsschwerpunkt. Allen Definitionen ist allerdings gemein, dass das Ergebnis der Dienstleistung eine gewisse Wirkung auf den Kunden hat. Vor diesem Hintergrund kommt (Buhl et al. 2008, S. 61) zu folgenden definitorischen Eigenschaften für Dienstleistungen:

- Intangibilität und Immaterialität

- Gleichzeitigkeit von Erstellung und Konsum (Uno-actu-Prinzip)

- Integration des Kunden (als externer Faktor) in den Erstellungsprozess

$\mathrm{Zu}$ ähnlichen Ergebnissen kommen auch (Chesbrough und Spohrer 2006; Rai und Sambamurthy 2006, S. 328).

Übertragen auf die Problemdomäne universitärer Lehre lässt sich dies dahingehend konkretisieren, dass zwischen Dozent und Studierendem im Rahmen des Lehrerbringungsprozesses eine Interaktion stattfindet mit dem Ziel des Erwerbs von Fakten- und Handlungswissen sowie bestimmten fachlich übergreifenden Kompetenzen auf Seiten der Studierenden. Das exakte Ergebnis ist dabei nur schwer messbar (gerade bei übergeordneten Kompetenzen) und hängt stark davon ab, was der Lernende als Kunde zum Prozess beiträgt.

Sowohl die Interaktion zwischen Dozent und Studierendem, als auch die Bereithaltung des Potenzials (worunter konkret Mitarbeiter, Softwarewerkzeuge sowie auch Lehr-Lern-Konzepte und Fachwissen des Dozenten fallen) sind jedoch mit einem hohen Aufwand auf Seiten des Lehranbieters verbunden. Die gezielte Gestaltung von Dienstleistungen muss damit sowohl Input- als auch Outputbetrachtungen vereinen. Bezogen auf Lehrdienstleistungen werden damit zwei Perspektiven zusammengeführt, die sonst als eher konkurrierend angesehen werden (Mohr und Krcmar 2005, S. 8): Die Sicht des ökonomischen Bildungscontrollings, welches vorwiegend auf Kosteneffizienz bedacht ist, und das pädagogische Bildungscontrolling, welches auf einen möglichst hohen Lernerfolg abzielt.

Beides zu vereinen bedeutet die Bestrebung, die Produktivität von Bildungsdienstleistungen zu steigern. Produktivität bezeichnet dabei das Verhältnis von Output (Zufriedenheit, Lernerfolg und Kompetenzerwerb) zu Input (dem Aufwand auf Seiten des Lehrenden). Eine Steigerung der Produktivität wird im Idealfall durch gleichzeitige Reduzierung des Inputs und Steigerung des Outputs erreicht. Als mögliche Stellschrauben für Ersteres kommen Standardisierung, Teilautomatisierung und gezieltes Auslagern einzelner Aktivitäten an Studierende in Frage. Eine Steigerung des Outputs dagegen wird in der Lehre u. a. durch eine erhöhte Individualisierung angestrebt. Obwohl gerade moderne IKT hier neue Möglichkeiten eröffnen, beides zu verbinden, schöpfen gegenwärtige Bildungsangebote dieses Potenzial nicht ausreichend aus (Gabriel et al. 2007, S. 5). Eine strukturierte 
Methode zur Modellierung von Dienstleistungen wie das Service Blueprinting kann jedoch helfen, die Problemstellung überschaubarer zu machen und Anhaltspunkte für Optimierungen zu liefern.

\subsection{Service Blueprinting}

Die Methode des Service Blueprinting geht zurück auf Arbeiten von Shostack (Shostack 1982, S. 49; Shostack 1984, S. 133) und wurde in zahlreichen Arbeiten fortentwickelt. (Zeithaml et al. 2006, S. 236ff) definieren das Service Blueprinting als eine Methode, die gleichzeitig den Dienstleistungsprozess, die Kundenschnittstellen und die Wahrnehmung der Dienstleisungserbringung aus Kundensicht darstellt. Ein Service Blueprint kann somit als zweidimensionales Bild eines Dienstleistungserstellungsprozesses angesehen werden: Die horizontale Achse stellt die Chronologie der einzelnen Prozessschritte dar, die sowohl vom Leistungserbringer als auch vom Kunden erbracht werden, und die vertikale Achse unterteilt die verschiedenen Stufen der Interaktion zwischen Dienstleistungserbringer und Kunden in Ebenen (Fließ und Kleinaltenkamp 2004, S. 396).

Obwohl sich die einzelnen Entwicklungsstufen und Anwendungsbeispiele des Service Blueprinting im Detail unterscheiden, haben sich verschiedene Ebenen durchgesetzt, die im Folgenden vorgestellt werden:

- „Line of interaction“: Diese Linie trennt Aktivitäten, die vom Kunden ausgeführt werden, von denen, die vom Dienstleister erbracht werden, und stellt selbst die Aktivitäten dar, die gemeinsam erbracht werden.

- „Line of visibility“: Hierbei wird unterschieden zwischen Aktivitäten (des Dienstleisters), die für den Kunden sichtbar respektive unsichtbar sind.

- „Line of internal interaction“: Hier findet eine Unterscheidung zwischen FrontOffice und Back-Office-Aktivitäten statt.

- „Line of implementation“: Diese trennt Management-Aktivitäten von Unterstützungsleistungen (z.B. IT-Systeme, technische Lösungen).

Je nach Industrie und Anwendungsbeispiel findet man weitere Abgrenzungen in der Literatur, wie z.B. die „Line of order penetration“ unterhalb der „Line of internal interaction". Auf diese wurde hier verzichtet, da die meisten nicht kundeninduzierten Aktivitäten von geringerem Aufwand auf Seiten des Lehrenden sind (bspw. Eintragung der Lehrveranstaltung im Prüfungssystem) bzw. ohnehin unterhalb der „Line of implementation“ liegen.

Die Stärke des Service Blueprinting Ansatzes ist es, dass neben der Modellierung des Leistungserstellungsprozess sowohl die Kommunikationsschnittstellen mit den Kunden sowie der Integrationsgrad in den Leistungserstellungsprozess detailliert dargestellt werden. Darauf aufbauend können diese entsprechend analysiert und optimiert bzw. Vorgaben für die Dienstleistungsgestaltung gewonnen werden. Die Methode des Service Blueprinting wurde bereits auf unterschiedlichste Dienstleistungen zu unterschiedlichen Zwecken angewandt (Fließ und Kleinalten- 
kamp 2004, S. 396). Im folgenden Abschnitt wird die Methode des Service Blueprinting auf den Kontext von Bildungsdienstleistungen angepasst.

\section{Service Blueprinting für Bildungsdienstleistungen}

Erste Betrachtungen zur strukturierten Analyse von Blended Learning unter Gesichtspunkten des Service Engineerings finden sich in (Gabriel et al. 2006, S. 76 90). Auch die Service Blueprinting Methode wurde bereits in der Lehre angewandt, allerdings unter einem anderen Fokus, dem der wahrgenommenen Servicequalität und Kundenzufriedenheit (Gabriel et al. 2008, S. 12). In unserer Arbeit werden jedoch dediziert auch Inputfaktoren berücksichtigt mit dem Ziel einer Steigerung der Produktivität.

Die dazu angestrebte Steigerung der Effizienz wird bei Dienstleistungen mittels verschiedener Formen von Standardisierungen (Stauss 2006, S. 325) und anderen gezielten Eingriffen erreicht, welche auf eine verbesserte Faktorkombination abzielen (Fließ und Kleinaltenkamp 2004, S. 398). Lernender und Lehrender sollen also ihre Ressourcen an Wissen, Methoden, Kompetenzen und Softwareprodukten derartig miteinander kombinieren, dass die Produktivität maximiert wird. Dazu lassen sich die einzelnen Teilaktivitäten eines Blueprints bspw. unter folgenden Gesichtspunkten analysieren:

Contact Point Analyse: Hierbei werden die Schnittstellen zwischen Lehrendem und Lernenden betrachtet. Probleme können dort aus mangelhaften Informationsflüssen resultieren.

Standardisierung von Kommunikationsschnittstellen: Die Schlussfolgerung einer Contact Point Analyse könnten Bestrebungen zur Standardisierung der Kommunikationsschnittstellen zwischen Lehrendem und Lernenden sein. Fragen sind pädagogisch erwünscht, eine schnelle Beantwortung durch den Lehrenden kann gerade in virtuellen Lernangeboten kritisch für die Motivation der Lernenden sein. Eine effektive Bearbeitung kann jedoch bspw. durch unnötig viele Kommunikationskanäle (Videochat, Forum, Text-Chat, E-Mail, ggf. Diskussionsseiten in einem Wiki, Telefon, persönliches Gespräch) erschwert werden, da hier u. a. Medienbrüche entstehen.

Integration der Kunden in den Leistungserstellungsprozess: Eine erhöhte Integration des Lernenden ist nicht zwangsläufig mit steigendem Aufwand für den Lehrenden verbunden. Stattdessen ist auch eine Auslagerung von Tätigkeiten an den Studierenden, die Verantwortungsübernahme im Sinne eines angeleiteten Selbststudiums, möglich.

Die Besonderheiten des Service Blueprintings für die Lehre werden an einem Fallbeispiel erläutert. Bei der nachfolgend betrachteten Lehrveranstaltung handelt es sich um die Vorlesung Einfübrung in die Wirtschaftsinformatike (Info1). Diese war ursprünglich eine traditionelle, teilnehmerstarke Lehrveranstaltung mit Tutorium, die um E-Learning Methoden ergänzt und so zu einer Blended Learning Veranstal- 
tung ausgebaut wurde. Als elektronische Lerninhalte wurden zunächst neben den Präsentationsfolien Videoaufzeichnungen der Lehrveranstaltung auf einer Lernplattform angeboten. In einem zweiten Schritt sollten dann Web Based Trainings (WBTs) zur Verfügung gestellt werden, welche der individuellen Nachbereitung und Wissensvertiefung in Ergänzung zu den Präsenzterminen dienen sollten. Die Rechtfertigung des Zusatzaufwands für die multimedialen Inhalte ergibt sich aus folgenden didaktischen Überlegungen:

- Unterschiedliche Lerntypen benötigen unterschiedliche Formen von Lernmaterial oder Lernumgebung, sei es der Vortrag durch einen Dozenten, Übungen oder multimediales Lernmaterial (Kahiigi et al. 2007, S. 2).

- Lernende erhalten durch multimediale Lernmaterialien die Möglichkeit, beliebige Teile der Lerninhalte noch einmal zu betrachten (Zhang et al. 2004, S. 79).

- Die Flexibilität von Zeit und Ort kann im traditionellen Vorlesungssaal nicht erreicht werden (Zhang et al. 2004, S. 76).

Die Darstellung der Vorlesung als Service Blueprint ist in der Abbildung $1 \mathrm{zu}$ finden. Bei der Realisierung möglicher Effizienzsteigerungspotenziale spielen die bereits erläuterten Ebenen des Blueprints eine wichtige Rolle: Sie zeigen an, wie stark der Lernende in eine Aktivität einbezogen wird, und wo deren Ergebnis wieder einfließt. Die einzelnen Linien haben im Fall der betrachteten Vorlesung folgende Bedeutungen:

- „Line of interaction“: Oberhalb dieser Linie befinden sich Tätigkeiten der Lernenden ohne Interaktion mit dem Lehrenden, also bspw. Selbstlernphasen. Unmittelbar auf der Linie befindliche, interaktive Aktivitäten wie Beratungsgespräche oder Präsenzveranstaltungen sind besonders schwer automatisierbar, tragen jedoch wesentlich zur wahrgenommenen Qualität der Lehre bei.

- „Line of visibility“: Für den Lernenden unsichtbare Dienstleistungen können im Vergleich zu interaktiven Tätigkeiten leichter angepasst werden, ohne dass damit eine wahrgenommene Qualitätsminderung einhergeht.

- „Line of internal interaction“: Eine Unterscheidung zwischen Front- und Backoffice-Aktivitäten macht in der Lehre nur begrenzt Sinn. Diese Linie wurde ursprünglich eingeführt, um die Komplexität des Dienstleistungserstellungsprozesses auf Dienstleisterseite zu reduzieren. Eine alternative Deutung dieser Linie für die Lehre findet sich in (Gabriel 2008, S. 8).

- „Line of implementation“: Hierunter liegen Unterstützungsaktivitäten wie die Bereitstellung der Lernplattform. Diese können zur Erzielung von Skaleneffekten oftmals durch Universitätseinrichtungen zentral erbracht werden. 


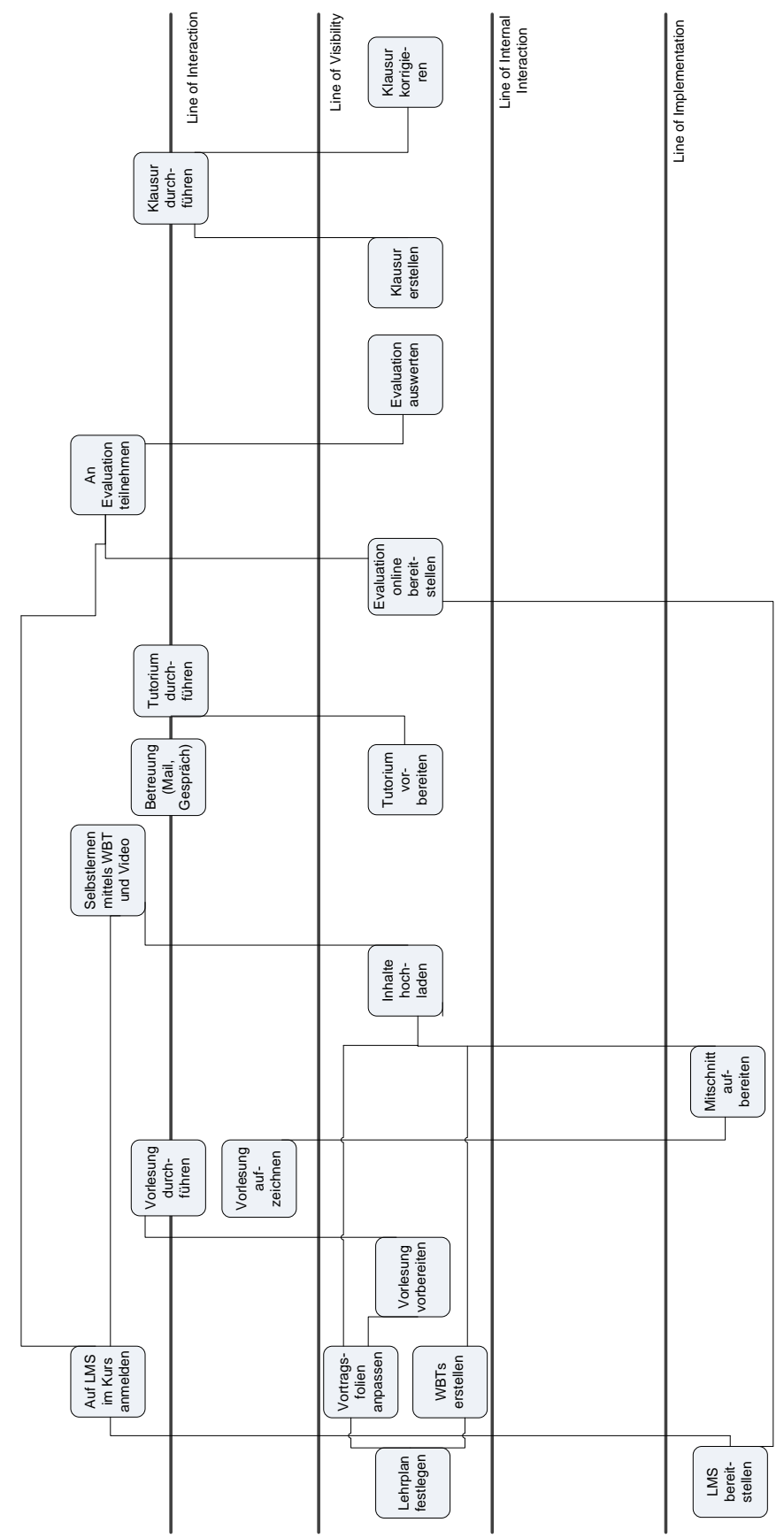

Abbildung 1: Service Blueprint der Lehrveranstaltung Info1 
Die rein lineare Darstellung des Blueprints dient der Vereinfachung, einzelne Aktivitäten wiederholen sich in der Realität oder folgen nicht exakt dieser chronologischen Ordnung, was für die weitere Betrachtung jedoch unwesentlich ist. Die dargestellten Teilaktivitäten lassen sich unter verschiedenen Fragestellungen betrachten: Lässt sich der Ablauf dieser Aktivität durch Standardisierung oder sogar Teilautomatisierung effizienter gestalten? Oder kann die Aktivität verstärkt an den Studierenden ausgelagert werden?

Bei dieser Betrachtung bietet es sich an, diejenigen Aktivitäten mit Priorität zu untersuchen, welche mit einem besonders hohen Aufwand für den Dozenten verbunden sind. Die Untersuchung des Beispiels Info1 ergab dabei folgende Einschätzung:

- Das Kurskonzept als solches existierte bereits und wird regelmäßig angepasst, der Hauptaufwand für die inhaltliche Planung ist somit bereits erledigt.

- Die Lernumgebung wird fachbereichsübergreifend zur Verfügung gestellt und gewartet, sodass an dieser Stelle keine Effizienzsteigerung zu erwarten ist.

- Die Vorbereitung des Tutoriums bietet ebenfalls wenig Spielraum für eine Reduzierung des Inputs.

- Der gesamte Klausurprozess kostet relativ viel Zeit, was jedoch in Anbetracht der Studierendenzahlen folgerichtig und gegenwärtig nicht vermeidbar ist.

- Die Betreuung der Studierenden kann ggf. durch definierte Schnittstellen und Verwendung von Werkzeugen wie einer FAQ verbessert und im Aufwand reduziert werden.

- Die Erstellung der WBTs wird als besonders aufwendig angesehen (vgl. z.B. Köhne 2005, S. 58f.) und hat sich auch in anderen Lehrveranstaltungen als äuBerst zeitintensiv dargestellt.

Aufgrund dieser Einschätzung wurde der effizienten Erstellung der WBTs die höchste Priorität eingeräumt. Im Gegensatz zu den ohnehin üblichen Folien müssen die WBTs weitestgehend selbsterklärend sein, die multimedialen Vorzüge des Mediums Computer ausnutzen und ein erhöhtes Maß an Interaktivität aufweisen. Da die Erstellung der WBTs somit den mit Abstand größten Teil des Aufwands für die Weiterentwicklung der Lehrveranstaltung ausmachen würde, haben wir uns in einem ersten Schritt darauf konzentriert, die Inhaltserstellung effizienter zu gestalten.

Das genaue Vorgehen bei der Erstellung der Inhalte wird nachfolgend erläutert und mit ersten empirischen Befunden gestützt.

\section{Umsetzung eines Fallbeispiels und erste empirische Befunde}

Die Erstellung hochwertiger Lernmaterialien, welche die Multimedialität aktueller IKT ausnutzen und so unterschiedliche Lerntypen ansprechen, sollte möglichst 
effizient durchgeführt werden. Eine mögliche Lösung für diese Aufgabe wurde innerhalb eines Pilotprojekts eingeführt, welches sich über zwei universitäre Lehrveranstaltungen erstreckt. Hierbei handelt es sich um die bereits genannte Info1 sowie eine weiterführende Veranstaltung zur Vermittlung grundlegender Kenntnisse in der Webprogrammierung (im Weiteren kurz Info2 genannt). Das Ziel bestand darin, Studierenden der Info1 umfassende multimediale Lernmaterialien zur Verfügung zu stellen, die ihnen helfen sollten, das in der Vorlesung Besprochene besser zu verinnerlichen. Aufgrund des geschilderten Aufwands jedoch wurden in diesen Prozess die Studierenden der Info2 integriert: Die Studierenden erlernen hier nicht nur Grundlagen der Webprogrammierung, sondern wenden diese aktiv an, indem sie selbst kleine Lerneinheiten zu Themen der Info1 entwickeln. Diese Materialien wiederum werden später nach Prüfung durch die Dozenten den Teilnehmern der Info1 zur Verfügung gestellt, um hier ein flexibles, selbstgesteuertes Lernen zu ermöglichen. Dieses Vorgehen trägt mehreren didaktischen Prinzipien Rechnung:

- „Lernen durch Lehren“: Der Perspektivwechsel hin zu einem Lehrenden überträgt den Studierenden Verantwortung und hilft ihnen, die zuvor bereits erlernten Inhalte nochmals zu vertiefen. Dieses didaktische Prinzip wurde bereits erfolgreich in Präsenzszenarien eingesetzt und fördert vor allem Kompetenzen im kommunikativen Bereich (Grzega und Schöner 2008, S. 171f).

- Relevante Aufgabenstellung: Die Studierenden wissen, dass ihre entwickelten Inhalte unmittelbar Anwendung finden und anderen Lernenden zu Gute kommen.

- Individuelle Selbstlerninhalte: Die zusätzlichen Selbstlerninhalte bieten den Lernenden der Info 1 eine weitere Auswahlmöglichkeit bei der Gestaltung ihrer eigenen Lernprozesse.

Über die genannte Methode wird die Individualisierung des Lernangebots verstärkt, ohne dass der Aufwand in zu hohem Maße wächst. Wichtig ist hierbei jedoch, dass die Studierenden nicht lediglich als „Lieferanten“ von Lerninhalten angesehen werden, sondern ihrerseits eine optimale Betreuung erhalten und nicht unter Effizienzbestrebungen leiden müssen. Die Befragung der Studierenden in der Veranstaltung Info 2 zeigt jedoch, dass diese das System durchaus schätzen (s. Tabelle 1).

So zeigt die Befragung hohe Werte für die generelle Zufriedenheit und insbesondere die Einschätzung der Praxisrelevanz der Veranstaltung. Daraus lässt sich schließen, dass die Methodik funktioniert, ohne für die betroffenen Studierenden mit Nachteilen verbunden zu sein. Im Gegenteil profitieren diese eher von dem erläuterten Konzept. 
Tabelle 1: Evaluierung

\begin{tabular}{|c|c|c|}
\hline Allgemeine Fragen & $\begin{array}{l}\text { Mittel- } \\
\text { wert* }\end{array}$ & $\begin{array}{c}\text { StdAb } \\
\text { w }\end{array}$ \\
\hline Wie zufrieden sind Sie mit der Veranstaltung generell? & 2,29 & 0,92 \\
\hline Wie würden Sie die Veranstaltung im Vergleich zu anderen bewerten? & 2,29 & 0,85 \\
\hline Wie innovativ finden Sie die Veranstaltung? & 1,76 & 0,66 \\
\hline Würden Sie die Veranstaltung an Kommilitonen weiterempfehlen? & 2,06 & 0,97 \\
\hline Wie zufrieden sind Sie mit dem Dozenten generell? & 2,00 & 0,79 \\
\hline \multicolumn{3}{|l|}{ Inhalt des Kurses } \\
\hline Wie zufrieden sind Sie mit dem Inhalt der Veranstaltung? & 2,41 & 0,94 \\
\hline Wie zufrieden sind Sie mit die Struktur der Veranstaltung? & 2,53 & 0,87 \\
\hline Wie bewerten Sie die Menge des gelernten Inhalts im Vergleich zum Arbeitsaufwand? & 2,94 & 1,03 \\
\hline Wie zufrieden sind Sie mit dem Schwierigkeitsgrad der Lehrveranstaltung? & 2,65 & 0,79 \\
\hline Wie zufrieden sind Sie mit der Menge des vermittelten Inhalts im Vergleich Zeit? & 2,71 & 0,85 \\
\hline Klarheit und Transparenz der Anforderungen an die Leistungserbringung & 2,53 & 1,23 \\
\hline Wie zufrieden sind Sie mit der Interaktivität der Veranstaltung? & 1,88 & 0,70 \\
\hline Wie zufrieden sind Sie mit der Praxisrelevanz der Veranstaltung? & 2,00 & 0,71 \\
\hline Wie zufrieden sind Sie mit den Begleitmaterialien zur Veranstaltung? & 2,71 & 1,05 \\
\hline \multicolumn{3}{|l|}{ Dozent } \\
\hline Der Dozent verfügt über das notwendige Fachwissen & 1,12 & 0,33 \\
\hline Die Erläuterungen des Dozenten sind wertvoll & 1,59 & 0,44 \\
\hline Der Dozent kann begeistern & 2,41 & 0,55 \\
\hline Der Dozent ist gut vorbereitet & 1,29 & 0,69 \\
\hline Der Dozent schafft es Interesse beim Studenten zu wecken & 2,35 & 0,57 \\
\hline Der Dozent ist sympathisch & 1,47 & 0,93 \\
\hline Der Dozent beantwortet Fragen klar und strukturiert & 1,76 & 0,60 \\
\hline Der Dozent steht auch außerhalb der Vorlesung für Fragen zur Verfügung & 2,06 & 0,79 \\
\hline \multicolumn{3}{|l|}{ Über mich } \\
\hline Wie stark ist Ihr Interesse am Inhalt / der Veranstaltung? & 1,82 & 0,73 \\
\hline Hat die Veranstaltung Ihr Interesse am Fachgebiet gefördert? & 2,53 & 0,80 \\
\hline Wie viel Aufwand haben Sie in diesen Kurs investiert? & 2,00 & 0,79 \\
\hline Wie stark haben Sie sich aktiv am Kurs beteiligt? & 1,59 & 0,87 \\
\hline
\end{tabular}

\section{Zusammenfassung und Ausblick}

Die vorliegende Arbeit stellt einen Ansatz zur produktivitätsorientierten Gestaltung von Lehrveranstaltungen vor. Hierzu wurde das Service Blueprinting angewandt, um ein Blended Learning Angebot in einzelne Aktivitäten zu zerlegen und $\mathrm{zu}$ analysieren. In einem ersten Schritt wurden gezielt Prozessschritte identifiziert, die besonders ressourcenintensiv sind. Darauf aufbauend wurde ein Fallbeispiel zur aktiven Integration von Studierenden in den Lehrmaterialerstellungsprozess vorgestellt und umgesetzt. Die ersten empirischen Ergebnisse zeigen, dass ein aus 
Lehrendensicht ressourcenschonenderes Vorgehen nicht zwangsläufig einen pädagogischen Nachteil bedeutet. Es ist sogar festzustellen, dass sich verschiedene Aspekte moderner Lehr-Lern-Szenarien und eine Senkung des Aufwands auf Seite der Lehrenden durchaus sinnvoll ergänzen und fördern können.

Für eine umfassende Produktivitätsbetrachtung muss in einem nächsten Evaluierungsschritt im kommenden Semester festgestellt werden, wie stark die Studierenden von den zusätzlichen multimedialen Lerninhalten profitieren.

Dennoch konnten bislang nur erste Schritte im Rahmen einer produktivitätsorientierten Gestaltung der Lehre durchgeführt und evaluiert werden. Zudem ist festzustellen, dass das Blueprinting zwar bei der Analyse der Ist-Situation hilft, jedoch die Entwicklung des Soll-Konzepts immer noch eine kreative Tätigkeit darstellt. Weitere Forschungsfragen können daher u. a. folgende Bereiche betreffen:

- Das Outsourcing weiterer Leistungen (sowohl didaktischer als auch ITDienstleistungen) an zentrale Einrichtungen der Universitäten

- Ermittlung empirischer Zahlen zum Aufwand einzelner Teilaktivitäten zur Verfeinerung des Blueprints

- Die Auslagerung weiterer Tätigkeiten an die Studierenden, u. a. durch Peer Reviews, Moderatorenrollen usw.

- Eine Contact Point Analyse, bspw. durch Klassifizierung eingehender Fragen der Studierenden, um Rückschlüsse auf generelle Probleme oder vorhandene Informationsdefizite zu ziehen.

Zudem liegt im Bereich der Dienstleistungsproduktivität noch hoher Forschungsbedarf. Neue Forschungsergebnisse hier im speziellen oder im Service Engineering allgemein bieten enormes Potenzial, um die universitäre Lehre zu verbessern.

\section{Literatur}

Brugger P, Stroh A, Schmidt P (2009) Hochschulen auf einen Blick. Statistisches Bundesamt, Wiesbaden.

Chesbrough H, Spohrer J (2006) A research manifesto for services science. Communications of the ACM 49 (7).

Fließ S, Kleinaltenkamp M (2004) Blueprinting the service company Managing service processes efficiently. Journal of Business Research 57 (4): 392-404.

Gabriel R, Gersch M, Weber P (2007) Mass Customization und

Serviceplattformstrategien im Blended Learning Engineering-konzeptionelle Grundlagen und evaluationsgestützte Erfahrungen. In: Oberweis A, Weinhardt C, Gimpel H, Koschmider A, Pankratius V, Schnizler B (Hrsg) Tagungsband der Internationalen Tagung Wirtschaftsinformatik, Karlsruhe. 
Gabriel R, Gersch M, Weber P (2008) Lern-Service-Blueprinting als Instrument einer am Lernprozess orientierten Messung von Zufriedenheit im Blended Learning. Tagungsband der Multikonferenz Wirtschaftsinformatik (MKWI), München.

Gabriel R, Gersch M, Weber P, Venghaus C (2006) Blended Learning Engineering: Der Einfluss von Lernort und Lernmedium auf Lernerfolg und Lernzufriedenheit - Eine evaluationsgestützte Untersuchung. In: Breitner M, Bruns B, Lehner F (Hrsg) Neue Trends im E-Learning. Physica, Heidelberg: $75-92$.

Grzega J, Schöner M (2008) The didactic model LdL (Lernen durch Lehren) as a way of preparing students for communication in a knowledge society. Journal of Education for Teaching 34 (3): 167 - 175.

Kahiigi E, Ekenberg L, Hansson M (2007) Exploring the e-Learning State of art. Proceedings of the Conference on E-Learning.

Köhne S (2005) Didaktischer Ansatz für das Blended Learning: Konzeption und Anwendung von Educational Patterns. Universität Hohenheim.

Kultusministerkonferenz (2005) Prognose der Studienanfänger, Studierenden und Hochschulabsolventen bis 2020. Kultusministerkonferenz, Bonn, http://www.kmk.org/fileadmin/veroeffentlichungen_beschluesse/2005/2005 _10_01-Studienanfaenger-Absolventen-2020.pdf Abruf am 2009-11-27.

Mohr M, Krcmar H (2005): Bildungscontrolling: State of the Art und Bedeutung für die IT-Qualifizierung, Garching b. München: Technische Universität München, Lehrstuhl für Wirtschaftsinformatik.

OECD (2003) The Sources of Economic Growth in OECD Countries. Organisation for Economic Co-Operation and Development, http://www.oecd.org/dac/ictcd/docs/otherdocs/OtherOECD_eco_growth. pdf Abruf am 20.09.2009.

Rai A, Sambamurthy V (2006) The Growth of Interest in Services Management: Opportunities for Information Systems Scholars. Information Systems Research 17 (4).

Shostack LG (1982) How to Design a Service. European Journal of Marketing 16 (1): 49-63.

Shostack LG (1984) Design Services that Deliver. Harvard Business Review (84115): 133-139.

Stauss B (2006) Plattformstrategie im Dienstleistungsbereich. In: Bullinger H-J, Scheer A-W (Hrsg) Service Engineering. Springer, Heidelberg: 321-340. 
Wissenschaftsrat (2006) Empfehlungen zum arbeitsmarkt- und demographiegerechten Ausbau des Hochschulsystems. Wissenschaftsrat, Berlin, http://www.wissenschaftsrat.de/texte/7083-06.pdf Abruf am 2009-1127

Zeithaml VA, Bitner M, Gremler DD (2006) Services marketing : integrating customer focus across the firm. McGraw-Hill/Irwin, Boston, 2006.

Zhang D, Zhao JL, Zhou L, Jay F. Nunamaker J (2004) Can e-learning replace classroom learning? Commun. ACM 47 (5): 75-79. 